\title{
Blomstrand lethal chondrodysplasia
}

INSERM

\section{Source}

INSERM. (1999). Orphanet: an online rare disease and orphan drug data base.

Blomstrand lethal chondrodysplasia. ORPHA:50945

Blomstrand lethal chondrodysplasia (BLC) is a neonatal osteosclerotic dysplasia (see this term) characterized by advanced endochondral bone maturation, very short limbs, dwarfism and prenatal lethality. 